\title{
La edad y sexo como factores condicionantes del control de enfermedad crónica en el primer nivel de atención: estudio retrospectivo
}

\author{
Jesús Alberto Martínez Mandujano, ${ }^{1,2, a, b, c}$ Tranquilina Gutiérrez Gómez, ${ }^{2, d}$ María Isabel Peñarrieta de Córdova, \\ Florabel Flores Barrios, ${ }^{2, \mathrm{f}}$ Rodrigo César León Hernández, ${ }^{1,2, \mathrm{~g}}$ María del Socorro Piñones Martínez. ${ }^{2, \mathrm{~d}}$
}

\begin{abstract}
Martínez MJA, Gutiérrez GT, Peñarrieta CMI, Flores BF, León HRC, Piñones MMS. La edad y sexo como factores condicionantes de control de enfermedad crónica en el primer nivel de atención: estudio retrospectivo. Cuid salud, jul-dic 2015; 2(2):213-219.
\end{abstract}

\section{RESUMEN}

Objetivo: determinar en usuarios diagnosticados con diabetes e hipertensión de un Programa de Crónicos, su estado de control por edad y sexo. Metodología: estudio retrospectivo, realizado sobre la base de datos del Sistema Integrado de Salud (SIS) de enero del 2012; con una población de 4037 usuarios, obtenida del Informe Mensual de Actividades Realizadas en una Unidad Médica SIS-SS-CE-H con énfasis en el Programa de Salud del Adulto y el Anciano. Resultados: usuarios diabéticos en tratamiento $76 \%$ (1287) y bajo control $24 \%$ (406). Usuarios hipertensos en tratamiento 67\% (1570) y en control 33\% (774). La edad no fue un factor de riesgo para un buen control de la Hipertensión (OR=0,8; IC: 0,8-1,15), tampoco para la Diabetes $(\mathrm{OR}=1,19$; IC: $0,9-1,5)$. El sexo no fue un factor de riesgo para tener un buen control en la Hipertensión (OR=0,8; IC: 0,671,02) tampoco en la Diabetes $(\mathrm{OR}=0,8$; IC: 0,7 1,14) al comparárselos con el grupo que presenta buen control de la enfermedad. Conclusiones: el porcentaje de usuarios en el grupo de tratamiento fue elevado, sin embargo, con deficiente control de la diabetes o hipertensión. El buen o mal control de la enfermedad no está relacionado con la edad ni el sexo.

Palabras clave: enfermedad crónica, atención primaria de salud, adherencia al tratamiento, enfermería.
Martínez MJA, Gutiérrez GT, Peñarrieta CMI, Flores BF, León HRC, Piñones MMS. Age and sex as determinants of chronic disease control in primary care: a retrospective study. Cuid salud, jul-dic 2015; 2(2):213-219.

\begin{abstract}
Objective: To determine in users diagnosed with diabetes and hypertension of a Chronic Program, their state of control by age and sex. Methodology: Retrospective study, based on data from the Integrated Health System (SIS) of January 2012; With a population of 4037 users, obtained from the Monthly Report of Activities carried out in a Medical Unit SIS-SS-CE-H with emphasis on the Health Program of Adult and Elderly. Results: Diabetic patients under treatment $76 \%$ (1287) and under control 24\% (406). Hypertensive patients under treatment 67\% (1570) and in control 33\% (774). Age was not a risk factor for good control of hypertension $(\mathrm{OR}=0.8$; CI: 0.8-1.15), neither for Diabetes $(\mathrm{OR}=1.19$; CI: 0.9- 1.5). Sex was not a risk factor for good control in Hypertension (OR $=0.8$; CI: 0.67-1.02) neither in Diabetes $(\mathrm{OR}=0.8$; CI: 0.7- 1,14) when compared to the group that has good control of the disease. Conclusions: The percentage of users receiving treatment was high, however, they have poor control of diabetes or hypertension. Good or bad control of the disease is not related to age or sex.
\end{abstract}

Keywords: Chronic illness, primary health care, adherence to treatment, nursing. 


\section{INTRODUCCIÓN}

En la actualidad, el aumento en la prevalencia de enfermedades crónicas y degenerativas en todo el mundo, como las enfermedades cardiovasculares, el cáncer, las enfermedades respiratorias crónicas y la diabetes, sin dejar de lado enfermedades neurológicas o reumáticas, además de alterar significativamente la calidad de vida, son la causa de alrededor del $60 \%$ de las muertes, de las cuales el $80 \%$ ocurren en países de bajos y medianos ingresos. ${ }^{1}$

Estudios de la Organización Mundial de la Salud (OMS) señalan que para 2020 el 75\% de las muertes en el mundo serán atribuibles a este tipo de enfermedades. ${ }^{2}$

La epidemia mundial de enfermedades crónicas no transmisibles causó en el 2005, unos 35 millones de defunciones, o sea $60 \%$ del total de los fallecimientos registrados a nivel mundial, $80 \%$ en los países de ingresos bajos y medianos y unos 16 millones entre personas menores de 70 años de edad. Está proyectado que para el 2015 morirán en el mundo 41 millones de personas por estas causas, quienes no cuentan con un programa de prevención y control. ${ }^{3}$ El logro del objetivo global de reducción de la tasa de mortalidad por enfermedades crónicas de $2 \%$ cada año, podría evitar la muerte de 36 millones de personas entre el 2005 y $2015 .^{4}$

México presenta una transición epidemiológica que se caracteriza por el predominio cada vez mayor de las enfermedades no transmisibles y las lesiones. A mitad del siglo pasado, alrededor de $50 \%$ de los decesos en el país se debían a infecciones comunes, problemas reproductivos y padecimientos asociados a la desnutrición. Hoy estas enfermedades concentran menos del $15 \%$ de los decesos, mientras que los padecimientos no transmisibles y las lesiones son responsables de poco menos de $75 \%$ y $11 \%$ de las muertes en el país, respectivamente. ${ }^{5}$

Entre las ECNT se encuentra la diabetes, que es una enfermedad crónica de causas múltiples, en su etapa inicial no produce síntomas y cuando se detecta tardíamente y no se trata adecuadamente ocasiona complicaciones de salud graves como infarto del corazón, ceguera, falla renal, amputación de las extremidades inferiores y muerte prematura. ${ }^{6}$ La Norma Oficial Mexicana y las Guías de Práctica Clínica de la Secretaría de
Salud definen a la diabetes mellitus como un conjunto de trastornos metabólicos que afecta a diferentes órganos y tejidos. ${ }^{7}$

Una persona diabética es aquella cuyas cifras de glucosa en sangre es igual o superior a $126 \mathrm{mg} / \mathrm{dl}$, con una hemoglobina glicosilada superior al $6,5 \%$ o aquella cuya glucemia en un test de sobrecarga oral de glucosa sea igual o superior a $200 \mathrm{mg} / \mathrm{dl} .^{8}$

La diabetes afecta actualmente a más de 366 millones de personas en el mundo, en México ocupa el primer lugar como causa de defunciones; en ambos sexos las tasas de mortalidad muestran una tendencia ascendente, con más de70 mil muertes y 400000 casos nuevos anuales. Según la Dirección General de Información en Salud, en el 2007 hubo más defunciones en las mujeres $(37,202$ muertes) que en los hombres $(33,310)$, con una tasa de 69,2 por 100000 habitantes en mujeres y de 64 en hombres, diferencias importantes a considerar en las acciones preventivas de detección, diagnóstico y tratamiento de este padecimiento. ${ }^{9}$

La diabetes mellitus tipo 2 (DM2) se caracteriza por hiperglucemias constantes, como resultado de una alteración en la producción y utilización de la insulina $10 \mathrm{y}$ produce complicaciones agudas y crónicas, las cuales se subdividen en microvasculares y macrovasculares. $\mathrm{Su}$ tratamiento se basa en el equilibrio de la alimentación, los fármacos y el ejercicio físico regular junto con una adecuada educación diabetológica, de manera que tanto el paciente como su familia dispongan de las habilidades y conocimientos necesarios para gestionar el día a día de la diabetes. ${ }^{11}$

Otra de las enfermedades igualmente de alta prevalencia en México y con gran impacto en la calidad de vida de las personas y sus familias cuando ésta no es bien tratada y controlada es la hipertensión arterial, su prevalencia en el país es de $30,05 \%$. Al igual que en otros países, la frecuencia de hipertensión se vincula con la edad, la obesidad y el género, siendo el segundo motivo de consulta en el primer nivel de atención. Sólo $19,4 \%$ de los usuarios hipertensos con tratamiento se encuentra bajo control, es decir, con cifras de presión arterial menores de 140/90 mmHg. ${ }^{12}$

El sobrepeso y la obesidad son factores predisponentes para el desarrollo de esta enfermedad y se estima que cerca del $70 \%$ en la 
población en México los padece. La hipertensión arterial es considerada un predictor de morbi mortalidad para enfermedades cardiovasculares, entre las que se destacan la enfermedad cerebrovascular, el infarto del miocardio, la insuficiencia cardiaca, la enfermedad arterial periférica y la insuficiencia renal. Destacando que la modificación de los factores de riesgo y el estilo de vida, disminuye su incidencia y favorece el control. $^{13}$

En México no se ha encontrado una evaluación de la efectividad de estos servicios de salud del primer nivel de atención con relación a identificar si los usuarios de dichos servicios presentan indicadores adecuados sobre la evolución de su enfermedad y cumplimiento del tratamiento.

Uno de los problemas registrados en personas con enfermedades como la hipertensión y la diabetes es la adherencia al tratamiento por parte de los pacientes, la importancia de este problema está dada por las repercusiones que tiene desde el punto de vista clínico, económico y psicosocial; además de su efecto en la calidad de la atención, la relación médico-paciente, entre otros.

Este estudio evaluó, de forma parcial, esta situación identificando el porcentaje de usuarios que se encuentran con indicadores de evolución negativa frente a su enfermedad (diagnosticados con diabetes e hipertensos) y analizando si la edad y el sexo son factores de riesgo para presentar esta condición verssus los usuarios que presentan indicadores positivos en la evolución de la enfermedad.

El sistema de registro en los centros de salud asigna a los usuarios al grupo de tratamiento cuando presentan indicadores negativos de la evolución de su enfermedad y, al grupo de control, cuando presentan indicadores positivos con relación a la evolución de su enfermedad (glucosa/hipertensión controlada, buena adherencia al tratamiento, sin complicaciones). Estos resultados son las primeras evidencias de evaluación sobre el programa en personas con padecimientos crónicos en los centros de salud de Tampico-México.

Los beneficiarios directos serán los usuarios de estos centros de salud, pues al mejorar la capacidad de respuesta por parte de los centros de salud a las necesidades presentadas por los usuarios se estaría contribuyendo a reducir complicaciones y mejorando su calidad de vida, disminuyendo la demanda a otros servicios de mayor complejidad como son los hosp.

\section{METODOLOGÍA}

El estudio fue retrospectivo, la población fue constituida por los usuarios registrados en el Informe Mensual de actividades realizadas en la Unidad Médica del Sistema Integrado de Salud de la Secretaria de Salud (SIS-SS-CE-H), con énfasis en el Programa de Salud del Adulto y el Anciano, incluyendo solamente las áreas de Diabetes mellitus e Hipertensión arterial, que se subdividen en dos grupos etarios (20 a 59 años y 60 años o más) seleccionando al total de usuarios registrados: 4037 usuarios del mes de enero del 2012.

El programa en estudio define los siguiente términos: Se considera paciente controlado con Diabetes mellitus aquel que se encuentra en tratamiento farmacológico o no farmacológico con niveles de glucosa plasmática o capilar en ayuno entre 70 y $130 \mathrm{md} / \mathrm{dl}$ o de hemoglobina glucosilada por debajo de 7\%. Se considera paciente controlado con Hipertensión arterial aquel que se encuentra en tratamiento no farmacológico o farmacológico, cuya presión arterial se encuentra en cifras menores a 149/90 según la Norma Oficial Mexicana NOM-015-SSA2-1994, para la prevención, tratamiento y control de la diabetes mellitus en la atención primaria. ${ }^{14}$

Por lo tanto, todo aquel paciente que se registre en el sistema SIS de acuerdo al resultado de la consulta en el mismo día que acude a recibirla en el rubro de "tratamiento" se refiere a que es un paciente que no lleva un control adecuado y el que se registre en "control" es aquel que ha llevado ya sea su control glicémico o sus cifras de presión arterial estables. A su vez, en cada uno de estos rubros se registran por edad y por sexo.

Los criterios de elegibilidad de la muestra fueron: ser usuario registrado por el Informe Mensual de Actividades Realizadas en la Unidad Médica SIS-SS-CE-H con énfasis en el Programa de Salud del Adulto y el Anciano, de las áreas de Diabetes Mellitus e Hipertensión Arterial, de 20 años a más durante el mes de enero del 2012.

Se utilizó la Razón de Momios o Odds Ratio para evaluar el riesgo de edad y sexo de presentar condiciones negativas en la evolución de su enfermedad versus estar en condiciones positivas en la evolución de su enfermedad. 
Los criterios utilizados por el sistema para ubicar al usuario en el grupo control (buena evolución de su enfermedad) fueron en el grupo de personas con diabetes mellitus; paciente que se encuentra con niveles de glucosa plasmática o capilar en ayuno entre 70 y 130 md/dl o de hemoglobina glucosilada por debajo de $7 \%$ se le ubica en el grupo de control, caso contrario se ubica en el grupo de tratamiento. En las personas con hipertensión arterial; paciente que su presión arterial se encuentra en cifras menores a 149/90 se le ubica en el grupo de control, caso contrario se ubica en el grupo de tratamiento.

Se registraron los datos de manera manual de la base de datos de registro del Informe Mensual de actividades realizadas en la Unidad Médica del Sistema integrado de Salud de la Secretaria de Salud (SIS-SS-CE-H) de la Jurisdicción de salud Nro. 2-Tampico. Una vez recolectados se vaciaron al Programa SPSS para el análisis estadístico correspondiente.

Se contó con la aprobación del comité de ética de la Facultad de Enfermería Tampico, Universidad Autónoma de Tamaulipas, así como del Comité de ética de la Jurisdicción de salud Nro. 2 donde se realizó el estudio.

\section{RESULTADOS}

La población de estudio quedó conformada por 1693 usuarios diabéticos y 2344 hipertensos. El total de la población según sexo fue 1060 hombres y 2977 mujeres y, por edad, 2033 se ubicaron entre 20 y 59 años mientras que 1700 tenían de 60 años a más. La distribución según diagnostico se presenta en la tabla 1 , donde se encuentra que 1693 fueron personas con diabetes y 2344 personas con hipertensión.

Tabla 1. Ubicación de usuario según diagnóstico y sexo.

\begin{tabular}{lccccc}
\hline \multirow{2}{*}{ Diagnóstico/grupo } & \multicolumn{2}{c}{ Hombres } & \multicolumn{2}{c}{ Mujeres } & \multirow{2}{*}{ Total } \\
& $\mathrm{N}$ & $\%$ & $\mathrm{~N}$ & $\%$ & \\
\hline Diabetes & 509 & 30 & 1184 & 70 & 1693 \\
Hipertensión & 551 & 24 & 1793 & 76 & 2344 \\
& & & & & \\
$\begin{array}{l}\text { Diabetes (edad en } \\
\text { años) }\end{array}$ & $20-59$ & & $\geq 60$ & & Total \\
$\begin{array}{l}\text { En grupo de } \\
\text { tratamiento }\end{array}$ & 827 & 77 & 460 & 74 & 1287 \\
$\begin{array}{l}\text { En grupo control } \\
\text { Hipertensión (edad }\end{array}$ & 244 & 23 & 162 & 26 & 403 \\
en años) & & & & & \\
$\begin{array}{l}\text { En grupo de } \\
\text { tratamiento }\end{array}$ & $20-59$ & & & & \\
En grupo control & 442 & 67 & 728 & 67 & 1570 \\
& 420 & 23 & 352 & 23 & 772 \\
\hline
\end{tabular}

Los resultados de la ubicación de usuarios con diabetes e hipertensión se describen en la tabla 1, donde se observa mayor porcentaje en ambos grupos de enfermedad en las mujeres, siendo $70 \%$ en la diabetes y $76 \%$ en la hipertensión.

Tabla.2 Evaluación de riesgo según edad y sexo.

\begin{tabular}{lcccc}
\hline \multirow{2}{*}{ Variables } & \multicolumn{2}{c}{ Diabetes } & \multicolumn{2}{c}{ Hipertensión } \\
\cline { 2 - 5 } & OR & IC & OR & IC \\
\hline Edad: & 1,19 & $0,9-1,5$ & 0,8 & $0,8-$ \\
$20-59$ vs $60 \geq$ & & & & 1,15 \\
Sexo: & 0,8 & $0,7-1,14$ & 0,8 & $0,67-$ \\
Hombres vs Mujer & & & & 1,02 \\
\hline
\end{tabular}

En la tabla 2, se describe los resultados de la evaluación de riesgo según edad y sexo para ambos grupos, encontrando que tanto la edad como el sexo no fueron factores de riesgo para presentar una evaluación negativa de su enfermedad, esto es pertenecer al grupo de tratamiento. Se encontró que el sexo es un factor protector en el grupo de hipertensos.

\section{DISCUSIÓN}

En el estudio se muestra que la mayoría de los usuarios se encuentra en el grupo de tratamiento, ya que del total de pacientes con diabetes e hipertensión corresponden al 76\% y $67 \%$ respectivamente; evidenciando que los pacientes no están llevando buen apego al tratamiento corroborando los resultados de previos estudios. ${ }^{15-17}$

Lo anterior indica la imperiosa necesidad que las instituciones de salud del primer nivel de atención busquen hacer los cambios correspondientes para obtener una mejor respuesta de la atención médica a los pacientes en los centros de salud, que contribuya a mejorar la evolución de la enfermedad crónica (diabetes mellitus e hipertensión arterial), pues los indicadores biológicos de control de la enfermedad muestran cifran arriba del estándar normal como glucosa, peso, presión arterial, así como el no cumplimiento de las indicaciones médicas. Asimismo, se estaría evitando que presenten menor riesgo de presentar complicaciones derivadas de un control adecuado como son las neuropatías, amputaciones para el caso de la diabetes y problemas circulatorios y cardiacos para el caso de hipertensión. ${ }^{18}$

En algunos estudios, se ha demostrado que en este nivel de atención, fortalecer el automanejo de la enfermedad por parte de los usuarios logra disminuir el uso de los servicios de salud, controlar 
el peso y una mejor adherencia al tratamiento, entre otras aspectos importantes que contribuyen a mejorar la calidad de vida de estas personas, ${ }^{19}$ proveer a los pacientes del conocimiento necesario, habilidades y la confianza (la autoeficacia) para tratar con los problemas relacionados de la enfermedad. ${ }^{20,21}$

En el estudio se encontró en los pacientes con diabetes que la edad no es un factor de riesgo para el control o no control de la enfermedad, con un OR: 1,19; IC: 0.9-1.5; resultado similar al de Mejía et al. ${ }^{12}$ que encontraron que la edad de 58 años y más como factor de riesgo para un mal control de la glucosa no mostró significancia. Cabe mencionar la dificultad de encontrar mayores estudios que permitan corroborar o evidenciar controversia si la edad es o no un factor de riesgo, aspecto también de gran importancia dado que la media de la población de fue de 99,6 usuarios con diabetes por centro de salud. Identificando un porcentaje importante en grupos de edad diferenciados entre adultos y ancianos, aspectos que se podría deducir también una diferencia en seguir un control adecuado o no.

Las edades de los usuarios de los centros de salud seleccionados en el estudio fueron de 20 a 59 años y de 60 años o más; con mayor porcentaje también en las mujeres $(73,7 \%)$. Según referencias, se encontró que el sexo podría ser un factor de riesgo para seguir un adecuado control de la enfermedad, con diferencias entre hombres y mujeres, pero es el hombre el que presenta mayor riesgo en el caso de la hipertensión. ${ }^{22}$

Los resultados del estudio permiten corroborar la no diferencia de riesgo entre hombres y mujeres, se muestra que el sexo no es factor de riesgo para estar en el grupo de tratamiento; a diferencia de Concha et al., ${ }^{15}$ quienes encontraron que el sexo femenino tiene mayor riesgo de presentar descompensación en comparación con los hombres, que tenía menor probabilidad de presentar procesos de descompensación. Por el contrario, Rodríguez ${ }^{22}$ demostró que uno de los factores no modificables para el mal control fue el sexo masculino.

Los resultados del estudio muestran que en pacientes con diabetes el sexo no es un factor de riesgo para tener un buen control de la diabetes, por lo contrario mantiene un valor de protección lo que se corrobora con el estudio de Mejía et al., ${ }^{12}$ quienes encontraron que el riesgo de descompensación en el sexo masculino no tiene significancia.
Esta controversia indica que aún hay que seguir investigando estas diferencias de riesgo en la población tampiqueña según sexo, pues esto permitirá focalizar mejor las intervenciones educativas con perspectiva de género, garantizando así mayor efectividad en los resultados de la atención médica.

Concluyendo, el porcentaje de usuarios que se encuentran en el grupo de tratamiento es elevado lo cual ha sido corroborado con distintos estudios y puede indicar que las instituciones de salud de primer nivel no están llevando a cabo las intervenciones pertinentes para que estos usuarios puedan llevar un control adecuado.

El riesgo de estar en el grupo de tratamiento no está relacionado con la edad de los individuos, es decir, no importa ser adulto o anciano, ya que pueden tener las mismas posibilidades de riesgo para estar en descontrol o no.

En los pacientes diabéticos e hipertensos no hay evidencias que relacionen el riesgo de presentar un mal control derivado por el sexo de las personas, pues tendrían la misma posibilidad de estar en descontrol o no. Es necesario complementar la base de datos del SIS incluyendo variables como hábitos alimenticios, actividad física y otras de autocuidado para facilitar seguimiento y evaluación.

Entre las limitaciones que se citan en el estudio destacan que los resultados solo podrán ser aplicados a la población estudiada, considerando que no hubo muestreo probabilístico, es necesario también llevar en cuenta otras variables para determinar con mayor precisión la pertenencia al grupo de tratamiento en el Programa de Crónicas, pues intervienen también en este proceso otras, entre ellos las comorbilidades, la continuidad regular en las consultas, el hecho de padecer hipertensión/diabetes simultáneamente, entre otros.

\section{Declaración de financiamiento y de conflictos de interés:}

El estudio fue financiado por los autores; declaran no tener conflictos de interés.

\section{Contribución de autoría:}

JAMM, TGG, MIPC, FFB, RCLH, MSPM: Concepción y diseño del estudio, recolección de los datos, análisis e interpretación de los resultados y aprobación de la versión que será publicada. 
Correspondencia:

María Isabel Peñarrieta de Córdova

Correo electrónico: decordova.maria.isabel@gmail.com

\section{REFERENCIAS BIBLIOGRÁFICAS}

1. World Health Organization. Preventing Chronic Deseases: A vital investment. Geneva: WHO, Public Health Agency of Canada; 2005. $182 \mathrm{p}$.

2. World Health Organization. Preventing chronic diseases: a vital investment: WHO Global Report. Geneva: 2005. 186p.

3. Strong KL, Mathers CD, Leeder S, Beaglehole R. Preventing chronic diseases: how many lives can we save? Lancet [serie de internet]. Oct 292005 [citado 20 de jun 2015]; 366:1578-82. Disponible en: http://www.thelancet.com/pdfs/journals/lancet/ PIIS0140-6736(05)67341-2.pdf

4. Abegunde DO, Mathers CD, Adam T, Ortegon M, Strong K. The burden and costs of chronic diseases in low-income and middle-income countries. Lancet [serie de internet]. Dic 08 2007 [citado 20 de mar 2014]; 370:1929-38. Disponible en: http://www.who.int/choice/publications/p_200 7_Chronic disease burden_Lancet.pdf

5. Secretaría de Salud. Programa Nacional de Salud 2007-2012. Por un México sano: construyendo alianzas para una mejor salud. México DF: Secretaría de Salud; 2007. 74p.

6. Hernández-Ávila M, Gutiérrez JP, ReynosoNoveron N. Diabetes mellitus en México. El estado de la epidemia. Salud pública Méx [serie de internet]. 2013 [citado 18 de may 2014]; 55(S2): S129-S136. Disponible en: http://www.scielo.org.mx/pdf/spm/v55s2/v55s $\underline{2 \mathrm{a} 9 . \mathrm{pdf}}$

7. Secretaría de Salud de México. Norma oficial Mexicana NOM-015-SSA2-1991 para la prevención, tratamiento y control de la diabetes. México DF: Secretaria de la Salud de México; 1991.

8. American Diabetes Association. Report of the Experte Committee on the Diagnosis and classification of Diabetes Mellitus. Diabetes Care [serie de internet]. Jan 2003 [citado 15 de may 2014]; 26 (S1): S5-S20. Disponible en: http://care.diabetesjournals.org/content/26/supp $\underline{11 / \text { s5.full-text.pdf }}$

9. Calvo I, Sánchez O, Yáñez A. Prevalencia de enfermedad renal crónica no diagnosticada en pacientes con diabetes mellitus tipo 2 en atención primaria a la salud. Med Int Méx [serie de internet]. 2015 [citado 12 de may 2014]; 31: 41-49. Disponible en: http://www.medigraphic.com/pdfs/medintmex/ $\underline{\text { mim-2015/mim151g.pdf }}$

10. López LHL, Pérez GRE, Monroy TR. Factores de riesgo y hábitos alimentarios en personas de 25 a 35 años, con y $\sin$ antecedentes de diabetes mellitus tipo 2. Rev Salud Pública y Nutr [serie de internet]. 2011 [citado 20 de jun 2014]; 12(2): [aproximadamente 10 laudas]. Disponible en: http://www.medigraphic.com/pdfs/revsalpubnu t/spn-2011/spn112e.pdf

11. Jansà M, Murillo S, Vidal M. Medical Nutritional Therapy and Therapeutic Patient Education in Diabetes. Rev ROL Enferm [serie de internet]. May 2011 [citado 12 de oct 2014]; 34 (5):16-23. Disponible en: https://www.researchgate.net/publication/5150 9448_Medical_nutritional therapy and therap eutic_patient_education_in_diabetes

12. Mejía-Rodríguez O, Paniagua-Sierra R, Valencia-Ortíz MR, Ruiz-García J, FigueroaNuéz B, Roa-Sánchez V. Factores relacionados con el descontrol de la presión arterial. salud pública de méxico [serie de internet]. Jul-ago 2009 [citado 10 de feb 2015]; 51(4):291-297. Disponible en: http://www.scielosp.org/pdf/spm/v51n4/v51n4 a05.pdf

13. Instituto Mexicano del Seguro Social. Diagnóstico y Tratamiento de la Hipertensión Arterial en el Primer Nivel de Atención. Evidencias y recomendaciones. México: Instituto Mexicano de Seguro Social; 2014. 76p. 
14. Secretaria de Salud. Estados Unidos Mexicanos. Modificación a la Norma Oficial Mexicana NOM-015-SSA2-1994, Para la prevención, tratamiento y control de la diabetes mellitus en la atención primaria para quedar como Norma Oficial Mexicana NOM-015SSA2-1994. Para la prevención, tratamiento y control de la diabetes [internet]. Secretaria de Salud: Mexico DF; 1999 [citado 25 de jul 2015]. [aproximadamente 10 laudas]. Disponible en: http://www.salud.gob.mx/unidades/cdi/nom/m $\underline{015 \mathrm{ssa} 24 . \mathrm{html}}$

15. Concha TMC, Rodríguez GCR. Funcionalidad familiar en pacientes diabéticos e hipertensos compensados y descompensados. Theoria [serie de internet]. 2010 [citado 10 de ago 2015]; 19(1):41-50. Disponible en: http://www.ubiobio.cl/miweb/webfile/media/19 4/v/v19-1/3.pdf

16. Lara EA, Aroch CA, Jiménez RA, Arceo GM, Velázquez MO. Grupos de ayuda mutua: estrategia para el control de diabetes e hipertensión arterial. Archivos de cardiología de México [serie de internet]. 2004 [citado 10 deb 215]; 74(4):330-336. Disponible en: http://novomania.com/wiki/pub/SaludInteligent e/GRUPOS_DE_AYUDA_MUTUA/ac0441.pd $\mathrm{f}$

17. Crespo N, Rosales E, Gonzales R, Crespo N, Hernández J. Caracterización de la diabetes mellitus. Rev Cubana Med Gen Integr [serie de internet]. Jul-ago 2003 [citado 12 de feb 2015]; 19(4): [aproximdamente 06 laudas]. Disponible en:

http://scielo.sld.cu/scielo.php?script=sci_arttext $\underline{\text { \&pid=S0864-21252003000400004 }}$

18. Córdova-Villalobos JA, Barriguete-Melendez JA, Lara-Esqueda A, Barquera S, Rosas-Peralta M, Hernández-Ávila M, León-May ME, Aguilar-Slinas C. Las Enfermedades Crónicas no transmisibles en México: Sinopsis Epidemiológica y prevención Integral. Salud pública Méx [serie de internet]. 2008 [citado 10 de mar 2015]; 50:419-427.Disponible en: http://www.scielosp.org/pdf/spm/v50n5/a15v5 0n5.pdf

19. Grady PA, Gough LL. El automanejo de las enfermedades crónicas: un método integral de atención. Am J Public Health [serie de internet]. 2014 [citado 10 de feb 2015]; 104:24-31. Disponible en: http://www.paho.org/journal/index.php?option =com docman\&view=download\&category slu $\mathrm{g}=$ pdfs-march-2015\&alias=872-el-automanejode-las-enfermedades-cronicas-un-metodo$\underline{\text { integral-de-atencion } \& \text { Itemid }=847}$

20. Lorig KR, Sobel DS, Ritter PL, Laurent D, Hobbs M. Effect of self-management program on patients with chronic disease. Eff Clin Pract. 2001; 4:256-262.

21. Ahn S, Basu R, Smith ML, Jiang L, Loring K, Withelaw N, Ory M. The impact of chronic disease self-management programs: healthcare savings through a community-based intervention. BMC Public Health [serie de internet]. 2013 [citado 10 de mar 2015]. Disponible en: https://www.ncbi.nlm.nih.gov/pmc/articles/PM C3878965/pdf/1471-2458-13-1141.pdf

22. Rodríguez HM. La actividad física en la prevención y tratamiento de la hipertensión arterial. InterSedes [serie de intrnet]. 2012 [citado 10 de feb 2014]; 13 (18): 142-156. Disponible en: http://www.redalyc.org/pdf/666/66624662008. pdf 